\title{
HIGH DEGREE PRECISION DECOMPOSITION METHOD FOR THE EVOLUTION PROBLEM WITH AN OPERATOR UNDER A SPLIT FORM
}

\author{
Zurab Gegechkori $^{1}$, Jemal Rogava ${ }^{2}$ and Mikheil Tsiklauri ${ }^{2}$
}

\begin{abstract}
In the present work the symmetrized sequential-parallel decomposition method of the third degree precision for the solution of Cauchy abstract problem with an operator under a split form, is presented. The third degree precision is reached by introducing a complex coefficient with the positive real part. For the considered schema the explicit a priori estimation is obtained.
\end{abstract}

Mathematics Subject Classification. 65M12, 65M15, 65M55.

Received: June 20, 2001. Revised: October 8, 2001.

\section{INTRODUCTION}

The study of the approximated schemas of a solution of evolution problems leads to the conclusion that to each approximated schema there corresponds a definite operator (solving operator of a discrete problem), which approximates a solving operator (semigroup) of a source continuous problem. The opposite is also true: constructing approximation of a continuous semigroup, we build an approximated schema of a solution of an evolution problem.

For example, if we apply Rotte's method for a solution of an evolution problem, a solving operator of the obtained difference problem will be a discrete semigroup and we come to a problem of approximating a continuous semigroup with the help of discrete semigroups (in this case see Kato [14], Chap. IX).

In case of applying a decomposition method, the solving operator of the applicable decomposed problem generates the Trotter formula [23], or the Chernoff formula [1,2], or a formula, which is a combination of these formulas. Therefore, the error estimation of a decomposition method is equivalent to a problem of approximating of a continuous semigroup using Trotter type formulas. Papers [12,18] (see also [19], Chap. II) are dedicated to the error estimations of Trotter type formulas.

The schema of decomposition, associated with the Trotter formula, allows us to split Cauchy problem for an evolution equation with an operator $A=A_{1}+A_{2}+\ldots+A_{m}$ to $m$ problems correspondingly with operators $A_{1}, A_{2}, \ldots, A_{m}$, which are solved sequentially on each time interval with the length $t / n$.

The decomposition schema, associated with the Chernoff formula, is known as a method of fractional steps (see Ianenko [11]).

Keywords and phrases. Decomposition method, Semigroup, Trotter formula, Cauchy abstract problem.

1 Iv. Javakhishvili Tbilisi State University, Tbilisi 380043, Georgia. e-mail: GegeZu.Cyber@viamnet.hepi.edu.ge

2 I. Vekua Institute of Applied Mathematics of Iv. Javakhishvili Tbilisi State University, Tbilisi 380043, Georgia. e-mail: JRogava@viam.hepi.edu.ge, MTsikla@viam.hepi.edu.ge 
As it is known, the decomposition method is sufficiently general for obtaining economical schemas for the solution of the multidimensional problems of mathematical physics. They can be divided into two groups: the schemas of sequential account (Ianenko [11], Samarskii [20], Marchuk [17], Samarskii and Vabishchevich [21], Fryazinov [5], Diakonov [4], Temam [22], Gordeziani [7]) and the schemas of parallel account (Gordeziani and Samarskii [10], Gordeziani and Meladze [8,9], Kuzyk and Makarov [16]). In [19] (see Chap. II) the explicit estimations for decomposition schemas of the parallel account are obtained, which were considered in [9]. At present, there are many works dedicated to the decomposition method (see $[17,21]$ ).

In the above-stated works the schemas considered are of the first or second degree precision. As far as we know, high degree precision decomposition formulas in case of two addends for the first time were obtained in $[3]$.

In the present work, a symmetrized sequential-parallel decomposition method of the third degree precision for the solution of the Cauchy abstract problem with operator $A=A_{1}+A_{2}+\ldots+A_{m}$, is presented. For the considered schema the explicit a priori estimation is obtained. Under explicit estimations we understand such a priori estimations for an error of solution, where the constants of a right member do not depend on a solution of an initial continuous problem, i.e. are absolute.

\section{Setting of the problem}

Let us consider the Cauchy abstract problem in the Banach space $X$ :

$$
\frac{\mathrm{d} u(t)}{\mathrm{d} t}+A u(t)=0, \quad t>0, \quad u(0)=\varphi
$$

Here $A$ is a closed linear operator with the domain $D(A)$, which is everywhere dense in $X, \varphi$ is a given element from $D(A)$.

Suppose that $(-A)$ operator generates a strongly continuous semigroup $\{\exp (-t A)\}_{t \geq 0}$, then the solution of the problem (1) is given by the following formula (see $[13,15])$ :

$$
u(t)=U(t, A) \varphi, \quad \varphi \in D(A),
$$

where $U(t, A) \equiv \exp (-t A)$ is a strongly continuous semigroup.

Let $A=A_{1}+A_{2}+\ldots+A_{m}$, where $A_{j} \quad(j=1,2, \ldots, m)$ are compactly defined, closed linear operators in $X$.

Let us introduce a difference net domain:

$$
\bar{\omega}_{\tau}=\left\{t_{k}=k \tau, k=1,2, \ldots, \tau>0\right\}
$$

Along with the problem (1) we consider two sequences of the following problems on each interval $\left[t_{k-1}, t_{k}\right]$ :

$$
\begin{array}{cc}
\frac{\mathrm{d} v_{k}^{1}(t)}{\mathrm{d} t}+\alpha A_{1} v_{k}^{1}(t)=0, & \frac{\mathrm{d} w_{k}^{1}(t)}{\mathrm{d} t}+\alpha A_{m} w_{k}^{1}(t)=0, \\
v_{k}^{1}\left(t_{k-1}\right)=u_{k-1}\left(t_{k-1}\right), & w_{k}^{1}\left(t_{k-1}\right)=u_{k-1}\left(t_{k-1}\right), \\
\frac{\mathrm{d} v_{k}^{2}(t)}{\mathrm{d} t}+\alpha A_{2} v_{k}^{2}(t)=0, & \frac{\mathrm{d} w_{k}^{2}(t)}{\mathrm{d} t}+\alpha A_{m-1} w_{k}^{2}(t)=0, \\
v_{k}^{2}\left(t_{k-1}\right)=v_{k}^{1}\left(t_{k}\right), & w_{k}^{2}\left(t_{k-1}\right)=w_{k}^{1}\left(t_{k}\right),
\end{array}
$$




$$
\begin{aligned}
& \frac{\mathrm{d} v_{k}^{m-1}(t)}{\mathrm{d} t}+\alpha A_{m-1} v_{k}^{m-1}(t)=0, \quad \frac{\mathrm{d} w_{k}^{m-1}(t)}{\mathrm{d} t}+\alpha A_{2} w_{k}^{m-1}(t)=0, \\
& v_{k}^{m-1}\left(t_{k-1}\right)=v_{k}^{m-2}\left(t_{k}\right), \quad w_{k}^{m-1}\left(t_{k-1}\right)=w_{k}^{m-2}\left(t_{k}\right), \\
& \frac{\mathrm{d} v_{k}^{m}(t)}{\mathrm{d} t}+A_{m} v_{k}^{m}(t)=0, \quad \frac{\mathrm{d} w_{k}^{m}(t)}{\mathrm{d} t}+A_{1} w_{k}^{m}(t)=0, \\
& v_{k}^{m}\left(t_{k-1}\right)=v_{k}^{m-1}\left(t_{k}\right), \quad w_{k}^{m}\left(t_{k-1}\right)=w_{k}^{m-1}\left(t_{k}\right), \\
& \frac{\mathrm{d} v_{k}^{m+1}(t)}{\mathrm{d} t}+\bar{\alpha} A_{m-1} v_{k}^{m-1}(t)=0, \quad \frac{\mathrm{d} w_{k}^{m+1}(t)}{\mathrm{d} t}+\bar{\alpha} A_{2} w_{k}^{m+1}(t)=0 \\
& v_{k}^{m+1}\left(t_{k-1}\right)=v_{k}^{m}\left(t_{k}\right), \quad w_{k}^{m+1}\left(t_{k-1}\right)=w_{k}^{m}\left(t_{k}\right), \\
& \frac{\mathrm{d} v_{k}^{2 m-2}(t)}{\mathrm{d} t}+\bar{\alpha} A_{2} v_{k}^{2 m-2}(t)=0, \quad \frac{\mathrm{d} w_{k}^{2 m-2}(t)}{\mathrm{d} t}+\bar{\alpha} A_{m-1} w_{k}^{2 m-2}(t)=0, \\
& v_{k}^{2 m-2}\left(t_{k-1}\right)=v_{k}^{2 m-3}\left(t_{k}\right), \quad w_{k}^{2 m-2}\left(t_{k-1}\right)=w_{k}^{2 m-3}\left(t_{k}\right), \\
& \frac{\mathrm{d} v_{k}^{2 m-1}(t)}{\mathrm{d} t}+\bar{\alpha} A_{1} v_{k}^{2 m-1}(t)=0, \quad \frac{\mathrm{d} w_{k}^{2 m-1}(t)}{\mathrm{d} t}+\bar{\alpha} A_{m} w_{k}^{2 m-1}(t)=0, \\
& v_{k}^{2 m-1}\left(t_{k-1}\right)=v_{k}^{2 m-2}\left(t_{k}\right), \quad w_{k}^{2 m-1}\left(t_{k-1}\right)=w_{k}^{2 m-2}\left(t_{k}\right) .
\end{aligned}
$$

Here $\alpha$ is a numerical complex parameter with $\operatorname{Re}(\alpha)>0, u_{0}(0)=\varphi$. Suppose that $\left(-A_{j}\right),\left(-\alpha A_{j}\right)$ and $\left(-\bar{\alpha} A_{j}\right)(j=1,2, \ldots, m)$ operators generate strongly continuous semigroups.

On each $\left[t_{k-1}, t_{k}\right](k=1,2, \ldots)$ interval $u_{k}(t)$ are defined as follows:

$$
u_{k}(t)=\frac{1}{2}\left[v_{k}^{2 m-1}(t)+w_{k}^{2 m-1}(t)\right]
$$

We consider the function $u_{k}(t)$ as an approximate solution of the problem (1) on the interval $\left[t_{k-1}, t_{k}\right]$.

The above-stated schema in case of $m=2$ addends is considered in [6].

We will need natural degrees of the operator $A=A_{1}+A_{2}+\ldots+A_{m} \quad\left(A^{s}, \quad s=2,3,4\right)$. In case of two addends $(m=2)$ they are defined as follows:

$$
\begin{gathered}
A^{2}=\left(A_{1}^{2}+A_{2}^{2}\right)+\left(A_{1} A_{2}+A_{2} A_{1}\right) \\
A^{3}=\left(A_{1}^{3}+A_{2}^{3}\right)+\left(A_{1}^{2} A_{2}+\ldots+A_{2}^{2} A_{1}\right)+\left(A_{1} A_{2} A_{1}+A_{2} A_{1} A_{2}\right) \\
A^{4}=\left(A_{1}^{4}+A_{2}^{4}\right)+\left(A_{1}^{3} A_{2}+\ldots+A_{2}^{3} A_{1}\right)+\left(A_{1}^{2} A_{2} A_{1}+\ldots+A_{2}^{2} A_{1} A_{2}\right)+\left(A_{1} A_{2} A_{1} A_{2}+A_{2} A_{1} A_{2} A_{1}\right) .
\end{gathered}
$$

Analogously are defined $A^{s}(s=2,3,4)$ when $m>2$. 
Obviously, the domain $D\left(A^{s}\right)$ of the operator $A^{s}$ is the intersection of the domains of its addends.

Let us introduce the following definitions:

$$
\begin{gathered}
\|\varphi\|_{A}=\left\|A_{1} \varphi\right\|+\ldots+\left\|A_{m} \varphi\right\|, \quad \varphi \in D(A), \\
\|\varphi\|_{A^{2}}=\sum_{i, j=1}^{m}\left\|A_{i} A_{j} \varphi\right\|, \quad \varphi \in D\left(A^{2}\right),
\end{gathered}
$$

where $\|\cdot\|$ is a norm in $X$, similarly are defined $\|\varphi\|_{A^{s}} \quad(s=3,4)$.

Theorem. Let the following conditions be satisfied:

(a) $\alpha=\frac{1}{2} \pm i \frac{1}{2 \sqrt{3}} \quad(i=\sqrt{-1})$;

(b) $\left(-\gamma A_{j}\right), \gamma=1, \alpha, \bar{\alpha} \quad(j=1,2, \ldots, m)$ and $(-A)$ operators generate strongly continuous semigroups, for which the following estimations hold correspondingly:

$$
\begin{gathered}
\left\|U\left(t, \gamma A_{j}\right)\right\| \leq \mathrm{e}^{\omega t}, \\
\|U(t, A)\| \leq M \mathrm{e}^{\omega t}, \quad M, \omega=\text { const }>0 ;
\end{gathered}
$$

(c) $U(s, A) \varphi \in D\left(A^{4}\right)$ for every fixed $s \geq 0$.

Then the following estimation holds:

$$
\left\|u_{k}\left(t_{k}\right)-u\left(t_{k}\right)\right\| \leq c \mathrm{e}^{\omega_{0} t_{k}} t_{k} \tau^{3} \sup _{s \in\left[0, t_{k}\right]}\|U(s, A) \varphi\|_{A^{4}}
$$

where $c, \omega_{0}$ are positive constants.

\section{Auxiliary lemma}

Let us prove the auxiliary lemma on which the proof of the theorem is based.

Lemma (see [6]). If the conditions (a) and (b) of the Theorem are satisfied and $m=2$, then

$$
\frac{1}{2}\left[U\left(\tau, \bar{\alpha} A_{1}\right) U\left(\tau, A_{2}\right) U\left(\tau, \alpha A_{1}\right)+U\left(\tau, \bar{\alpha} A_{2}\right) U\left(\tau, A_{1}\right) U\left(\tau, \alpha A_{2}\right)\right]=I-\tau A+\frac{1}{2} \tau^{2} A^{2}-\frac{1}{6} \tau^{3} A^{3}+R_{4}^{(2)}(\tau)
$$

where the following estimation holds for $R_{4}^{(2)}(\tau)$ :

$$
\left\|R_{4}^{(2)}(\tau) \varphi\right\| \leq c \mathrm{e}^{\omega_{0} \tau} \tau^{4}\|\varphi\|_{A^{4}}, \quad \varphi \in D\left(A^{4}\right) .
$$

Here $c, \omega_{0}$ are positive constants.

Proof. According to the formula (see Kato [14], p. 603):

$$
A \int_{r}^{t} U(s, A) \mathrm{d} s=U(r, A)-U(t, A), \quad 0 \leq r \leq t
$$


we can get the following expansion:

$$
U(t, A)=\sum_{i=0}^{k-1}(-1)^{i} \frac{t^{i}}{i !} A^{i}+R_{k}(t, A)
$$

where

$$
R_{k}(t, A)=(-A)^{k} \int_{0}^{t} \int_{0}^{s_{1}} \ldots \int_{0}^{s_{k-1}} U(s, A) \mathrm{d} s \mathrm{~d} s_{k-1} \ldots \mathrm{d} s_{1} .
$$

Let us consider the operator in the left side of equality (5). Let us decompose both its items from right to left according to the formula (7), so that each residual member is of the fourth degree. Then, using elementary algebraic transformations, we will get the right side of equality $(5)$, where for the residual member $R_{4}^{(2)}(\tau)$ the following presentation is true:

$$
R_{4}^{(2)}(\tau)=\frac{1}{2}\left[R_{1,2}(\tau)+R_{2,1}(\tau)\right]
$$

where

$$
\begin{aligned}
R_{i, j}(\tau)= & R_{4}\left(\tau, \bar{\alpha} A_{i}\right)-\tau R_{3}\left(\tau, \bar{\alpha} A_{i}\right) A_{j}+\frac{1}{2} \tau^{2} R_{2}\left(\tau, \bar{\alpha} A_{i}\right) A_{j}^{2}-\frac{1}{6} \tau^{3} R_{1}\left(\tau, \bar{\alpha} A_{i}\right) A_{j}^{3} \\
& +U\left(\tau, \bar{\alpha} A_{i}\right) R_{4}\left(\tau, A_{j}\right)-\alpha \tau R_{3}\left(\tau, \bar{\alpha} A_{i}\right) A_{i}+\alpha \tau^{2} R_{2}\left(\tau, \bar{\alpha} A_{i}\right) A_{j} A_{i}-\frac{1}{2} \alpha \tau^{3} R_{1}\left(\tau, \bar{\alpha} A_{i}\right) A_{j}^{2} A_{i} \\
& -\alpha \tau U\left(\tau, \bar{\alpha} A_{i}\right) R_{3}\left(\tau, A_{j}\right) A_{i}+\frac{1}{2} \alpha^{2} \tau^{2} R_{2}\left(\tau, \bar{\alpha} A_{i}\right) A_{i}^{2}-\frac{1}{2} \alpha^{2} \tau^{3} R_{1}\left(\tau, \bar{\alpha} A_{i}\right) A_{j} A_{i}^{2} \\
& +\frac{1}{2} \alpha^{2} \tau^{2} U\left(\tau, \bar{\alpha} A_{i}\right) R_{2}\left(\tau, A_{j}\right) A_{i}^{2}-\frac{1}{6} \alpha^{3} \tau^{3} R_{1}\left(t, \bar{\alpha} A_{i}\right) A_{i}^{3}-\frac{1}{6} \alpha^{3} \tau^{3} U\left(\tau, \bar{\alpha} A_{i}\right) R_{1}\left(\tau, A_{j}\right) A_{i}^{3} \\
& +U\left(\tau, \bar{\alpha} A_{i}\right) U\left(\tau, A_{j}\right) R_{4}\left(\tau, \alpha A_{i}\right), \quad i, j=1,2
\end{aligned}
$$

Hence, according to the formula (8) and condition (b) of the Theorem we obtain the estimation (6).

\section{Proof of the theorem}

Let us get back to the proof of the Theorem.

It is obvious, that according to the formula (2) for the system (3) we have:

$$
\begin{gathered}
v_{k}^{j}\left(t_{k}\right)=U\left(\tau, \alpha A_{j}\right) v_{k}^{j-1}\left(t_{k}\right), \quad j=1,2, \ldots, m-1, \\
v_{k}^{m}\left(t_{k}\right)=U\left(\tau, A_{m}\right) v_{k}^{m-1}\left(t_{k}\right), \\
v_{k}^{m+j}\left(t_{k}\right)=U\left(\tau, \bar{\alpha} A_{m-j}\right) v_{k}^{m+j-1}\left(t_{k}\right), \quad j=1,2, \ldots, m-1,
\end{gathered}
$$

where $k=1,2, \ldots$,

$$
v_{k}^{0}\left(t_{k}\right)=u_{k-1}\left(t_{k-1}\right), \quad u_{0}(0)=\varphi
$$

Hence we have:

$$
v_{k}^{2 m-1}\left(t_{k}\right)=V_{1}(\tau) u_{k-1}\left(t_{k-1}\right)
$$


where

$$
V_{1}(\tau)=U\left(\tau, \bar{\alpha} A_{1}\right) \ldots U\left(\tau, \bar{\alpha} A_{m-1}\right) U\left(\tau, A_{m}\right) U\left(\tau, \alpha A_{m-1}\right) \ldots U\left(\tau, \alpha A_{1}\right)
$$

Analogously we obtain that:

$$
w_{k}^{2 m-1}\left(t_{k}\right)=V_{2}(\tau) u_{k-1}\left(t_{k-1}\right)
$$

where

$$
V_{2}(\tau)=U\left(\tau, \bar{\alpha} A_{m}\right) \ldots U\left(\tau, \bar{\alpha} A_{2}\right) U\left(\tau, A_{1}\right) U\left(\tau, \alpha A_{2}\right) \ldots U\left(\tau, \alpha A_{m}\right) .
$$

So according to the formula (4) we obtain:

$$
u_{k}\left(t_{k}\right)=V(\tau) u_{k-1}\left(t_{k-1}\right)=V^{k}(\tau) \varphi
$$

where

$$
V(\tau)=\frac{1}{2}\left(V_{1}(\tau)+V_{2}(\tau)\right)
$$

Remark. The operator $V^{k}(\tau)$ is a solving operator of the above considered decomposed problem. It is obvious that according to the condition of the Theorem $\left(U\left(t, \gamma A_{i}\right) \leq \mathrm{e}^{\omega t}\right)$

$$
\left\|V^{k}(\tau)\right\| \leq \mathrm{e}^{\omega_{1} t_{k}}
$$

where $\omega_{1}=(2 m-1) \omega$. From here it follows the stability of the above-stated decomposition schema on each finite time interval.

Let us suppose that $W(\tau)$ is a combination (sum, product) of semigroups, generated by operators $\left(-\gamma A_{i}\right)$ $(i=1,2, \ldots, m)$. Let us decompose all semigroups including in the operator $W(\tau)$ according to the formula $(7)$, multiply these decompositions, group together the similar members and define the coefficients of the members $\left(-\tau A_{i}\right),\left(\tau^{2} A_{i} A_{j}\right)$ and $\left(\tau^{3} A_{i} A_{j} A_{k}\right)(i, j, k=1,2, \ldots, m)$ to be correspondingly $[W(\tau)]_{i},[W(\tau)]_{i, j}$ and $[W(\tau)]_{i, j, k}$ in the obtained decomposition.

If we decompose all semigroups in the $V(\tau)$ from right to left according to the formula (7) so that each residual member is of the fourth degree, we get the following formula:

$$
V(\tau)=I-\tau \sum_{i=1}^{m}[V(\tau)]_{i} A_{i}+\tau^{2} \sum_{i, j=1}^{m}[V(\tau)]_{i, j} A_{i} A_{j}-\tau^{3} \sum_{i, j, k=1}^{m}[V(\tau)]_{i, j, k} A_{i} A_{j} A_{k}+R_{4}^{(m)}(\tau) .
$$

Similarly to $R_{4}^{(2)}$, according to the first inequality of the condition (b) of the theorem the following estimation is true for $R_{4}^{(m)}(\tau)(m>2)$ :

$$
\left\|R_{4}^{(m)}(\tau) \varphi\right\| \leq c \mathrm{e}^{\omega_{2} \tau} \tau^{4}\|\varphi\|_{A^{4}}, \quad \varphi \in D\left(A^{4}\right)
$$

where $c, \omega_{2}$ are positive constants. 
It is obvious that:

$$
\begin{gathered}
{[V(\tau)]_{i}=\frac{1}{2}\left(\left[V_{1}(\tau)\right]_{i}+\left[V_{2}(\tau)\right]_{i}\right), \quad i=1,2, \ldots, m,} \\
{[V(\tau)]_{i, j}=\frac{1}{2}\left(\left[V_{1}(\tau)\right]_{i, j}+\left[V_{2}(\tau)\right]_{i, j}\right), \quad i, j=1,2, \ldots, m,} \\
{[V(\tau)]_{i, j, k}=\frac{1}{2}\left(\left[V_{1}(\tau)\right]_{i, j, k}+\left[V_{2}(\tau)\right]_{i, j, k}\right), \quad i, j, k=1,2, \ldots, m .}
\end{gathered}
$$

Let us compute coefficients $\left[V_{1}(\tau)\right]_{i}$. Obviously, we get the corresponding members of these coefficients from decomposition of only those multipliers (semigroups) of the operator $V_{1}(\tau)$ which are generated by operators $\left(-\gamma A_{i}\right)$. From decomposition of other semigroups only first addends (identical operators) will be used. So we have:

$$
\left[V_{1}(\tau)\right]_{i}=\left[U\left(\tau, A_{i}\right)\right]_{i}=1 .
$$

Analogously

$$
\left[V_{2}(\tau)\right]_{i}=\left[U\left(\tau, A_{i}\right)\right]_{i}=1 .
$$

So we have

$$
[V(\tau)]_{i}=1, \quad i=1,2, \ldots, m
$$

Let us compute coefficients $\left[V_{1}(\tau)\right]_{i, j}$. Obviously, we get the corresponding members of these coefficients from decomposition of only those multipliers (semigroups) of the operator $V_{1}(\tau)$ which are generated by operators $\left(-\gamma A_{i}\right)$ and $\left(-\gamma A_{j}\right)$. From decomposition of other semigroups only first addends (identical operators) will be used. So we have:

$$
\left[V_{1}(\tau)\right]_{i, j}=\left[U\left(\tau, \bar{\alpha} A_{i_{1}}\right) U\left(\tau, A_{i_{2}}\right) U\left(\tau, \alpha A_{i_{1}}\right)\right]_{i, j}
$$

Analogously

$$
\left[V_{2}(\tau)\right]_{i, j}=\left[U\left(\tau, \bar{\alpha} A_{i_{2}}\right) U\left(\tau, A_{i_{1}}\right) U\left(\tau, \alpha A_{i_{2}}\right)\right]_{i, j},
$$

where $\left(i_{1}, i_{2}\right)$ is a pair of $i$ and $j$ indices, arranged in an increasing order. According to the lemma we have:

$$
\frac{1}{2}\left(\left[U\left(\tau, \alpha A_{i_{1}}\right) U\left(\tau, A_{i_{2}}\right) U\left(\tau, \bar{\alpha} A_{i_{1}}\right)\right]_{i, j}+\left[U\left(\tau, \alpha A_{i_{2}}\right) U\left(\tau, A_{i_{1}}\right) U\left(\tau, \bar{\alpha} A_{i_{2}}\right)\right]_{i, j}\right)=\frac{1}{2} .
$$

So we have

$$
[V(\tau)]_{i, j}=\frac{1}{2}, \quad i, j=1,2, \ldots, m
$$

Let us compute coefficients $\left[V_{1}(\tau)\right]_{i, j, k}$. Obviously, we get the corresponding members of these coefficients from decomposition of only those multipliers (semigroups) of the operator $V_{1}(\tau)$, which are generated by operators $\left(-\gamma A_{i}\right),\left(-\gamma A_{j}\right)$ and $\left(-\gamma A_{k}\right)$. From decomposition of other semigroups only first addends (identical operators) will be used. So we have:

$$
\left[V_{1}(\tau)\right]_{i, j, k}=\left[U\left(\tau, \bar{\alpha} A_{i_{1}}\right) U\left(\tau, \bar{\alpha} A_{i_{2}}\right) U\left(\tau, A_{i_{3}}\right) U\left(\tau, \alpha A_{i_{2}}\right) U\left(\tau, \alpha A_{i_{1}}\right)\right]_{i, j, k}
$$


Analogously

$$
\left[V_{2}(\tau)\right]_{i, j, k}=\left[U\left(\tau, \bar{\alpha} A_{i_{3}}\right) U\left(\tau, \bar{\alpha} A_{i_{2}}\right) U\left(\tau, A_{i_{1}}\right) U\left(\tau, \alpha A_{i_{2}}\right) U\left(\tau, \alpha A_{i_{3}}\right)\right]_{i, j, k}
$$

where $\left(i_{1}, i_{2}, i_{3}\right)$ is a triple of $i, j$ and $k$ indices, arranged in an increasing order.

Firstly let us consider the case when $i=j=k$, we have:

$$
\left[V_{1}(\tau)\right]_{i, j, k}=\left[U\left(\tau, A_{i}\right)\right]_{i, i, i}=\frac{1}{6}
$$

and

$$
\left[V_{2}(\tau)\right]_{i, j, k}=\left[U\left(\tau, A_{i}\right)\right]_{i, i, i}=\frac{1}{6}
$$

Now let us consider the case when only two of $i, j, k$ indices are different. In this case we have:

$$
\left[V_{1}(\tau)\right]_{i, j, k}=\left[U\left(\tau, \bar{\alpha} A_{i_{1}}\right) U\left(\tau, A_{i_{2}}\right) U\left(\tau, \alpha A_{i_{1}}\right)\right]_{i, j, k}
$$

and

$$
\left[V_{2}(\tau)\right]_{i, j, k}=\left[U\left(\tau, \bar{\alpha} A_{i_{2}}\right) U\left(\tau, A_{i_{1}}\right) U\left(\tau, \alpha A_{i_{2}}\right)\right]_{i, j, k} .
$$

where $\left(i_{1}, i_{2}\right)$ is pair of different indices of $i, j$ and $k$ triple, arranged in an increasing order. According to the lemma we have:

$$
[V(\tau)]_{i, j, k}=\frac{1}{6}
$$

Now let us consider the case when $i, j, k$ indices are different. We have six variants. Let us consider each one separately:

Case 1. If $i<j<k$, then

$$
\begin{aligned}
{\left[V_{1}(\tau)\right]_{i, j, k} } & =\left[U\left(\tau, \bar{\alpha} A_{i}\right) U\left(\tau, \bar{\alpha} A_{j}\right) U\left(\tau, A_{k}\right) U\left(\tau, \alpha A_{j}\right) U\left(\tau, \alpha A_{i}\right)\right]_{i, j, k} \\
& =\left[U\left(\tau, \bar{\alpha} A_{i}\right)\right]_{i}\left[U\left(\tau, \bar{\alpha} A_{j}\right)\right]_{j}\left[U\left(\tau, A_{k}\right)\right]_{k}=\bar{\alpha}^{2}
\end{aligned}
$$

and

$$
\begin{aligned}
{\left[V_{2}(\tau)\right]_{i, j, k} } & =\left[U\left(\tau, \bar{\alpha} A_{k}\right) U\left(\tau, \bar{\alpha} A_{j}\right) U\left(\tau, A_{i}\right) U\left(\tau, \alpha A_{j}\right) U\left(\tau, \alpha A_{k}\right)\right]_{i, j, k} \\
& =\left[U\left(\tau, A_{i}\right)\right]_{i}\left[U\left(\tau, \alpha A_{j}\right)\right]_{j}\left[U\left(\tau, \alpha A_{k}\right)\right]_{k}=\alpha^{2} .
\end{aligned}
$$

So we have

$$
[V(\tau)]_{i, j, k}=\frac{1}{2}\left(\alpha^{2}+\bar{\alpha}^{2}\right)=\frac{1}{6} .
$$

Case 2. If $i<k<j$, then

$$
\begin{aligned}
{\left[V_{1}(\tau)\right]_{i, j, k} } & =\left[U\left(\tau, \bar{\alpha} A_{i}\right) U\left(\tau, \bar{\alpha} A_{k}\right) U\left(\tau, A_{j}\right) U\left(\tau, \alpha A_{k}\right) U\left(\tau, \alpha A_{i}\right)\right]_{i, j, k} \\
& =\left[U\left(\tau, \bar{\alpha} A_{i}\right)\right]_{i}\left[U\left(\tau, A_{j}\right)\right]_{j}\left[U\left(\tau, \alpha A_{k}\right)\right]_{k}=\alpha \bar{\alpha}
\end{aligned}
$$


and

$$
\left[V_{2}(\tau)\right]_{i, j, k}=\left[U\left(\tau, \bar{\alpha} A_{j}\right) U\left(\tau, \bar{\alpha} A_{k}\right) U\left(\tau, A_{i}\right) U\left(\tau, \alpha A_{k}\right) U\left(\tau, \alpha A_{j}\right)\right]_{i, j, k}=0
$$

So we have

$$
[V(\tau)]_{i, j, k}=\frac{1}{2} \alpha \bar{\alpha}=\frac{1}{6}
$$

Case 3. If $j<i<k$, then

$$
\left[V_{1}(\tau)\right]_{i, j, k}=\left[U\left(\tau, \bar{\alpha} A_{j}\right) U\left(\tau, \bar{\alpha} A_{i}\right) U\left(\tau, A_{k}\right) U\left(\tau, \alpha A_{i}\right) U\left(\tau, \alpha A_{j}\right)\right]_{i, j, k}=0
$$

and

$$
\begin{aligned}
{\left[V_{2}(\tau)\right]_{i, j, k} } & =\left[U\left(\tau, \bar{\alpha} A_{k}\right) U\left(\tau, \bar{\alpha} A_{i}\right) U\left(\tau, A_{j}\right) U\left(\tau, \alpha A_{i}\right) U\left(\tau, \alpha A_{k}\right)\right]_{i, j, k} \\
& =\left[U\left(\tau, \bar{\alpha} A_{i}\right)\right]_{i}\left[U\left(\tau, A_{j}\right)\right]_{j}\left[U\left(\tau, \alpha A_{k}\right)\right]_{k}=\alpha \bar{\alpha} .
\end{aligned}
$$

So we have

$$
[V(\tau)]_{i, j, k}=\frac{1}{2} \alpha \bar{\alpha}=\frac{1}{6}
$$

Case 4. If $j<k<i$, then

$$
\left[V_{1}(\tau)\right]_{i, j, k}=\left[U\left(\tau, \bar{\alpha} A_{j}\right) U\left(\tau, \bar{\alpha} A_{k}\right) U\left(\tau, A_{i}\right) U\left(\tau, \alpha A_{k}\right) U\left(\tau, \alpha A_{j}\right)\right]_{i, j, k}=0
$$

and

$$
\begin{aligned}
{\left[V_{2}(\tau)\right]_{i, j, k} } & =\left[U\left(\tau, \bar{\alpha} A_{i}\right) U\left(\tau, \bar{\alpha} A_{k}\right) U\left(\tau, A_{j}\right) U\left(\tau, \alpha A_{k}\right) U\left(\tau, \alpha A_{i}\right)\right]_{i, j, k} \\
& =\left[U\left(\tau, \bar{\alpha} A_{i}\right)\right]_{i}\left[U\left(\tau, A_{j}\right)\right]_{j}\left[U\left(\tau, \alpha A_{k}\right)\right]_{k}=\alpha \bar{\alpha} .
\end{aligned}
$$

So we have

$$
[V(\tau)]_{i, j, k}=\frac{1}{2} \alpha \bar{\alpha}=\frac{1}{6}
$$

Case 5. If $k<i<j$, then

$$
\begin{aligned}
{\left[V_{1}(\tau)\right]_{i, j, k} } & =\left[U\left(\tau, \bar{\alpha} A_{k}\right) U\left(\tau, \bar{\alpha} A_{i}\right) U\left(\tau, A_{j}\right) U\left(\tau, \alpha A_{i}\right) U\left(\tau, \alpha A_{k}\right)\right]_{i, j, k} \\
& =\left[U\left(\tau, \bar{\alpha} A_{i}\right)\right]_{i}\left[U\left(\tau, A_{j}\right)\right]_{j}\left[U\left(\tau, \alpha A_{k}\right)\right]_{k}=\alpha \bar{\alpha}
\end{aligned}
$$

and

$$
\left[V_{2}(\tau)\right]_{i, j, k}=\left[U\left(\tau, \bar{\alpha} A_{j}\right) U\left(\tau, \bar{\alpha} A_{i}\right) U\left(\tau, A_{k}\right) U\left(\tau, \alpha A_{i}\right) U\left(\tau, \alpha A_{j}\right)\right]_{i, j, k}=0
$$

So we have

$$
[V(\tau)]_{i, j, k}=\frac{1}{2} \alpha \bar{\alpha}=\frac{1}{6}
$$


Case 6. If $k<j<i$, then

$$
\begin{aligned}
{\left[V_{1}(\tau)\right]_{i, j, k} } & =\left[U\left(\tau, \bar{\alpha} A_{k}\right) U\left(\tau, \bar{\alpha} A_{j}\right) U\left(\tau, A_{i}\right) U\left(\tau, \alpha A_{j}\right) U\left(\tau, \alpha A_{k}\right)\right]_{i, j, k} \\
& =\left[U\left(\tau, A_{i}\right)\right]_{i}\left[U\left(\tau, \alpha A_{j}\right)\right]_{j}\left[U\left(\tau, \alpha A_{k}\right)\right]_{k}=\alpha^{2}
\end{aligned}
$$

and

$$
\begin{aligned}
{\left[V_{2}(\tau)\right]_{i, j, k} } & =\left[U\left(\tau, \bar{\alpha} A_{i}\right) U\left(\tau, \bar{\alpha} A_{j}\right) U\left(\tau, A_{k}\right) U\left(\tau, \alpha A_{j}\right) U\left(\tau, \alpha A_{i}\right)\right]_{i, j, k} \\
& =\left[U\left(\tau, \bar{\alpha} A_{i}\right)\right]_{i}\left[U\left(\tau, \bar{\alpha} A_{j}\right)\right]_{j}\left[U\left(\tau, A_{k}\right)\right]_{k}=\bar{\alpha}^{2} .
\end{aligned}
$$

So we have

$$
[V(\tau)]_{i, j, k}=\frac{1}{2}\left(\alpha^{2}+\bar{\alpha}^{2}\right)=\frac{1}{6}
$$

Finally, for any triple $(i, j, k)$ we have:

$$
[V(\tau)]_{i, j, k}=\frac{1}{6}
$$

Inserting in (11) the obtained coefficients, we will get:

$$
\begin{aligned}
V(\tau) & =I-\tau \sum_{i=1}^{m} A_{i}+\frac{1}{2} \tau^{2} \sum_{i, j=1}^{m} A_{i} A_{j}-\frac{1}{6} \tau^{3} \sum_{i, j, k=1}^{m} A_{i} A_{j} A_{k}+R_{4}^{(m)}(\tau) \\
& =I-\tau \sum_{i=1}^{m} A_{i}+\frac{1}{2} \tau^{2}\left(\sum_{i=1}^{m} A_{i}\right)^{2}-\frac{1}{6} \tau^{3}\left(\sum_{i=1}^{m} A_{i}\right)^{3}+R_{4}^{(m)}(\tau) \\
& =I-\tau A+\frac{1}{2} \tau^{2} A^{2}-\frac{1}{6} \tau^{3} A^{3}+R_{4}^{(m)}(\tau) .
\end{aligned}
$$

According to the formula (7) we have:

$$
U(\tau, A)=I-\tau A+\frac{1}{2} \tau^{2} A^{2}-\frac{1}{6} \tau^{3} A^{3}+R_{4}(\tau, A)
$$

According to the second inequality of the condition (b) of the theorem the following estimation is true for $R_{4}(\tau, A)$ :

$$
\left\|R_{4}(\tau, A) \varphi\right\| \leq c \mathrm{e}^{\omega \tau} \tau^{4}\left\|A^{4} \varphi\right\| \leq c \mathrm{e}^{\omega \tau} \tau^{4}\|\varphi\|_{A^{4}}
$$

According to the formulas (13) and (14) we have:

$$
U(\tau, A)-V(\tau)=R_{4}(\tau, A)-R_{4}^{(m)}(\tau)
$$

Hence using inequalities (12) and (15) we can get the following estimation:

$$
\|[U(\tau, A)-V(\tau)] \varphi\| \leq c \mathrm{e}^{\omega_{2} \tau} \tau^{4}\|\varphi\|_{A^{4}} .
$$


According to the formulas (2) and (9) we have:

$$
\begin{aligned}
u\left(t_{k}\right)-u_{k}\left(t_{k}\right) & =\left[U\left(t_{k}, A\right)-V^{k}(\tau)\right] \varphi=\left[U^{k}(\tau, A)-V^{k}(\tau)\right] \varphi \\
& =\sum_{i=1}^{k} V^{k-i}(\tau)[U(\tau, A)-V(\tau)] U((i-1) \tau, A) \varphi .
\end{aligned}
$$

Hence according to the inequalities (10) and (16) we can obtain the following estimation:

$$
\begin{aligned}
\left\|u\left(t_{k}\right)-u_{k}\left(t_{k}\right)\right\| & \leq \sum_{i=1}^{k}\|V(\tau)\|^{k-i}\|[U(\tau, A)-V(\tau)] U((i-1) \tau, A) \varphi\| \\
& \leq \sum_{i=1}^{k} \mathrm{e}^{\omega_{1}(k-i) \tau} c \mathrm{e}^{\omega_{2} \tau} \tau^{4}\|U((i-1) \tau, A) \varphi\|_{A^{4}} \\
& \leq c \mathrm{e}^{\omega_{0} t_{k}} \tau^{4} \sum_{i=1}^{k}\|U((i-1) \tau, A) \varphi\|_{A^{4}} \\
& \leq c \mathrm{e}^{\omega_{0} t_{k}} t_{k} \tau^{3} \sup _{s \in\left[o, t_{k}\right]}\|U(s, A) \varphi\|_{A^{4}} .
\end{aligned}
$$

\section{Conclusion}

In the case when operators $A_{1}, A_{2}, \ldots, A_{m}$ are matrices, it is obvious that conditions of the theorem are automatically satisfied. Also conditions of the theorem are satisfied, if $A_{1}, A_{2}, \ldots, A_{m}$ and $A$ are self-adjoint, positive definite operators. The requirement $\alpha A$ operator $\left(\alpha=1 / \sqrt{3}\left(\cos 30^{\circ}+i \sin 30^{\circ}\right)\right)$ must generate a strongly continuous semigroup puts the condition for the spectrum of $A$. Namely, the spectrum of $A$ must be placed within sector with the angle less than 120 degrees, because in case of turning of spectrum by \pm 30 degrees (this is caused by multiplying of $A$ on $\alpha$ parameter) the spectrum area will stay in the positive (right) half-plane.

Third degree precision is reached by introducing a complex parameter. Because of this, each equation of the given decomposed system is changed by a pair of real equations, unlike lower degree precision schemas. To solve the specific problem, (for example) the matrix factorization may be used, where the coefficients are the matrices of the second order, unlike lower degree precision schemas, where the common factorization may be used.

It must be noted that the sum of the absolute values of coefficients of the addends of transition operator $V(\tau)$ equals to one, unlike the high degree precision decomposition schemas considered in [3]. Hence, the considered schema is stable for any bounded operators $A_{1}, A_{2}, \ldots, A_{m}$.

\section{REFERENCES}

[1] P.R. Chernoff, Note on product formulas for operators semigroups. J. Funct. Anal. 2 (1968) 238-242.

[2] P.R. Chernoff, Semigroup product formulas and addition of unbounded operators. Bull. Amer. Mat. Soc. 76 (1970) 395-398.

[3] B.O. Dia and M. Schatzman, Commutateurs de certains semi-groupes holomorphes et applications aux directions alternées. RAIRO Modél. Math. Anal. Numér. 30 (1996) 343-383.

[4] E.G. Diakonov, Difference schemas with decomposition operator for Multidimensional problems. JNM and MPh 2 (1962) 311-319.

[5] I.V. Fryazinov, Increased precision order economical schemas for the solution of parabolic type multidimensional equations. JNM and MPh 9 (1969) 1319-1326.

[6] Z.G. Gegechkori, J.A. Rogava and M.A Tsiklauri, Sequential-Parallel method of high degree precision for Cauchy abstract problem solution. Tbilisi, in Reports of the Enlarged Session of the Seminar of I. Vekua Institute of Applied Mathematics $\mathbf{1 4}$ (1999). 
[7] D.G. Gordeziani, On application of local one dimensional method for solving parabolic type multidimensional problems of 2m-degree. Proc. Acad. Sci. GSSR 3 (1965) 535-542.

[8] D.G. Gordeziani and H.V. Meladze, On modeling multidimensional quasi-linear equation of parabolic type by one-dimensional ones. Proc. Acad. Sci. GSSR 60 (1970) 537-540.

[9] D.G. Gordeziani and H.V. Meladze, On modeling of third boundary value problem for the multidimensional parabolic equations of an arbitrary area by one-dimensional equations. JNM and MPh 14 (1974) 246-250.

[10] D.G. Gordeziani and A.A. Samarskii, Some problems of plates and shells thermo elasticity and method of summary approximation. Complex Anal. Appl. (1978) 173-186.

[11] N.N. Ianenko, Fractional steps method of solving multidimensional problems of mathematical physics. Nauka, Novosibirsk (1967) $196 \mathrm{p}$.

[12] T. Ichinose and S. Takanobu, The norm estimate of the difference between the Kac operator and the Schrodinger semigroup. Nagoya Math. J. 149 (1998) 53-81.

[13] K. Iosida, Functional analysis. Springer-Verlag (1965).

[14] T. Kato, The theory of perturbations of linear operators. Mir, Moscow (1972) $740 \mathrm{p}$.

[15] S.G. Krein, Linear equations in Banach space. Nauka, Moscow (1971), 464 p.

[16] A.M. Kuzyk and V.L. Makarov, Estimation of exactitude of summarized approximation of a solution of the Cauchy abstract problem. RAN USSR 275 (1984) 297-301.

[17] G.I. Marchuk, Split methods. Nauka, Moscow (1988) 264 p.

[18] J.A. Rogava, On the error estimation of Trotter type formulas in the case of self-Adjoint operator. Funct. Anal. Appl. 27 (1993) 84-86.

[19] J.A. Rogava, Semi-discrete schemas for operator differential equations. Tbilisi, Georgian Technical University press (1995) $288 \mathrm{p}$.

[20] A.A. Samarskii, Difference schemas theory. Nauka, Moscow (1977), 656 p.

[21] A.A. Samarskii and P.N. Vabishchevich, Additive schemas for mathematical physics problems. Nauka, Moscow (1999).

[22] R. Temam, Quelques méthodes de décomposition en analyse numérique. Actes Congrés Intern. Math. (1970) 311-319.

[23] H. Trotter, On the product of semigroup of operators. Proc. Amer. Mat. Soc. 10 (1959) 545-551.

To access this journal online:

www.edpsciences.org 